\title{
Outcomes of Femoro-Popliteal Vascular Trauma Surgery: A Three-Year Survey (2011-2013) in Ahvaz Golestan Hospital (Iran)
}

\author{
Iraj Nazari ${ }^{1}$, Seyed Mansour Alamshah ${ }^{1}$, \\ Seyed Masoud Moosavi ${ }^{3}$ and Mohammadreza Mashhadi ${ }^{4 *}$ \\ ${ }^{1}$ Vascular Surgeon, General Surgery Department, Ahvaz Jundishapur \\ University of Medical Sciences, Ahvaz, Iran. \\ ${ }^{2}$ General Surgeon, General Surgery Department, Ahvaz Jundishapur \\ University of Medical Sciences, Ahvaz, Iran. \\ ${ }^{3}$ General Surgeon, General Surgery Department and Fellowship of Trauma, \\ Ahvaz Jundishapur University of Medical Sciences, Ahvaz, Iran.
}

DOI: http://dx.doi.org/10.13005/bbra/1891

(Received: 20 July 2015; accepted: 04 September 2015)

\begin{abstract}
Popliteal artery injury is one of the peripheral vascular injuries, is a major cause of disability to maintain the damaged limb, and orthopedic injuries are its most common cause. This study aimed to investigate the effects of femoro-popliteal vascular injuries surgery and discover probable defects and weaknesses during the evaluation and treatment of patients to mitigate the effects of disability. This descriptive and analytical study retrospectively and prospectively evaluates the outcomes of femoro-popliteal vascular trauma surgery in fifty-four patients referred to Ahvaz Golestan Hospital during September 2011 to May 2013. The patients were evaluated for average time of ischemia, trauma mechanism, combined damages, vascular repair techniques, postoperative complications, recovery rate of fasciotomies, rate of recovery, and amputation rate. The data were analyzed using the independent $t$-test and Fisher's exact test and $P<0.05$ was considered as significant. The mean age of the 44 patients was $27.7 \pm 12$ years that $86.4 \%$ of them were men and $13.6 \%$ were female and $72.7 \%$ of patients were referred from the different counties of Khuzestan province to this center and the rest were from the provincial capital, Ahvaz. In $63.6 \%$ of patients, trauma mechanism was blunt and in the rest was penetrating. The most common combined injuries were bone fractures, and the most common vascular repair method was the saphenous vein interposition graft. Furthermore, $40.9 \%$ of victims underwent a fasciotomy that $72.2 \%$ were fully recovered. The average time of ischemia (from incident to reperfusion) was $26.1 \pm 24.6 \mathrm{hrs}$. Although the majority of subjects $(\mathbf{7 2 . 7 2 \% )}$ was referred from the various cities of the province and caused to longer average time ischemia (from the accident until reperfusion), recovery rate after vascular repair surgery was $88.6 \%$, and only five patients $(\mathbf{1 1 . 4 \% )}$ were amputated. Therefore, the long time average of ischemia (from the accident until reperfusion) alone cannot cause vascular remodeling or failure to perform an amputation. The multiple combined damages, including damage to the vein could have an important role in the amputation of a limb so that vein damage ratio did no show significant differences in two amputee and recovered groups $(p=0.008)$.
\end{abstract}

Key words: Popliteal artery injury, Prognosis, Disability

Among all the peripheral artery traumas, popliteal artery injury is one of the main causes of

\footnotetext{
* To whom all correspondence should be addressed. Tel.:/Fax: +986133743076; Mob.: +989161135185; E-mail: iraj.nazari@yahoo.com
}

the disability to maintain the damaged limb and known as one of the most threatening peripheral vascular injuries that can make a serious risk to the life of the lower extremities².

The orthopedic injury is the most common cause of this phenomenon (80\%), so that it can be 
seen in 30\% of the posterior knee dislocation cases and in Supracondylar femur fractures or in tibial plateau and also in fractures of combined fibula tibia $^{3}$ that 12 to $50 \%$ have been reported according to the type and amount of the fracture displacement ${ }^{4}$.

The cause of damage following arterial trauma can be the intimal flap, thrombosis, rupture and the spasms ${ }^{5}$. Its result is the injury of acute ischemia popliteal artery and if immediate action is not taken at the right time for it, leads to amputation or severe long-term complications such as loss of sensation and complications related to $\mathrm{it}^{3}$.

Lower limb artery damage often is accompanied by numerous regional pathologies (combined) bone, vein, soft tissue and nerve. Management of such patients who are also with numerous topical injuries simultaneously is challenging because, in order to maintain the performance of the limbs the blood supply should be performed for a member suffering from ischemia within six to eight hours ${ }^{4,6}$.

To establish blood recirculation in the limbs in order to prevent permanent damage a time less than six hours has been usually accepted for ischemia; however, it also depends other factors such as the level of the damage, previous arterial disease, trauma to the soft tissues, vein damage, and previous lower extremity surgery ${ }^{5}$ ).

The prevalence of popliteal vascular damage differs in various medical centers and up to $31 \%$ has also been reported among patients with lower limb artery damage, mainly following penetrant traumas $(81 \% \text { of cases) })^{2,7}$. Patients with clear symptoms of lower limb ischemia should undergo surgery and arterial exploration ${ }^{1}$.

The likelihood of cut off a limb after popliteal vascular damage in keeping with cases such as blunt trauma, a long ischemia time, simultaneous bone and muscle damage, and simultaneous artery and vein damage increase considerably 8 . In cases where the time of ischemia is stretched more than 12 hours and organs are irreversible in the clinical examinations the initial amputation should be in mind ${ }^{9}$.

In this study, we decided to evaluate the consequences of the results of femoro-popliteal vascular surgeries in the lower extremity trauma, and detect the possible defects and weaknesses in the assessment and treatment of patients and finally tempt to eliminate them in order to reduce the postoperative complications and birth defects. Some reasons for performing this study are as follows: the sample size limitation in the similar studies, significant outbreaks of lower extremity traumas; secondary vascular damage; serious and permanent side effects caused by it; a lack of study of results of femoro-popliteal vascular surgeries in the southwest of the country and this premise that a considerable part of these victims referred to the trauma center of the Ahvaz Golestan Hospital, are from the city fringe areas and the surrounding counties and therefore consequences can be potentially different from urban trauma patients ${ }^{10}$.

\section{MATERIALSAND METHODS}

This descriptive-analytic study was conducted retrospectively and prospectively to evaluate the outcomes of femoro-popliteal vascular trauma surgery in Ahvaz Golestan hospital. This study was approved by Ethical Committee of Ahvaz Jundishapur University of Medical Sciences (Eth Ref. No. AJUMS.Rec.1392.64 92.4.15). In this study all patients who underwent the emergency femoropopliteal vascular surgery from September 2011 to May 2013 (for one year) by referring to the Archive of the Center, their files were extracted and questionnaires concerning each patient were completed. Also, patients who underwent the emergency femoro-popliteal vascular surgery from September 2012 to May 2013 (for nine months), at the same time of hospitalization, were follow-up and questionnaires concerning each patient was completed. Then, all patients who had passed at least two months since the surgery, were invited by telephone to assess their recovery status and underwent the clinic clinical reexamination by two vascular surgical and fellowship trauma specialty physicians in Golestan hospital, and a questionnaire was completed.

In terms of the trauma mechanism of, vascular damage type, combined damages (damage of the bone and nerve-soft tissue and vein), the patients were evaluated. Also they were evaluated for hemodynamic status on arrival to the emergency room, the time of the accident until 
referring to the emergency room, and referring to the emergency room until the time of surgery; in addition, an evaluation of ischemia time (the time of the accident until the surgery), vascular restoration procedures were performed where lateral repair, end to end anastomosis, saphenous interposition grafts, and saphenous bypass graft were performed.

Furthermore, clinical examinations of the affected foot before and after surgery during hospitalization in terms of pain, the distal pulses, C.F, warmth of the limb, laceration of the leg, posterior knee ecchymosis, sense and moving the foot, and compartment syndrome symptoms, need for fasciotomy, infection, necrosis of the muscles, cases and the count of debridement was conducted and finally, amputations were evaluated and recorded on the questionnaire.

Data were finally entered into SPSS version 19 and independent t-test and Fisher's exact test were used for statistical analysis and $\mathrm{P}<0.05$ was considered as significance level.

\section{RESULTS}

The mean age of 44 patients was $27.7 \pm 12$ years, with a minimum age of 4 years and a maximum age of 70 years that 38 people $(86.4 \%)$ of the patients were male and six persons (13.6\%) were female. In terms of the hemodynamic status of patients on admission to the emergency room, 41 people (93.2\%) and three patients (6.8\%) were stable and unstable, respectively.

Of the total patients only two persons were with underlying comorbidity (diabetes, ischemic heart disease) and only one person of the referred patients before the arrival of the emergency, have had heparin injections.

Of the 44 patients examined 32 patients (72.72\%) had been deployed from the center of Khuzestan Province's counties and the rest were from the provincial capital, Ahvaz County.

The mechanism of trauma in 28 patients (63.6\%) was blunt and in the 16 patients (36.4\%) was penetrant. Of patients with penetrating trauma two persons have been due to Stab wound, and 14 people have been due to gunshot or shotgun. Twenty-seven patients (61.4\%) of 44 patients examined were with posterior knee ecchymosis and 25 (56.1\%) of the patient were with comorbid skin

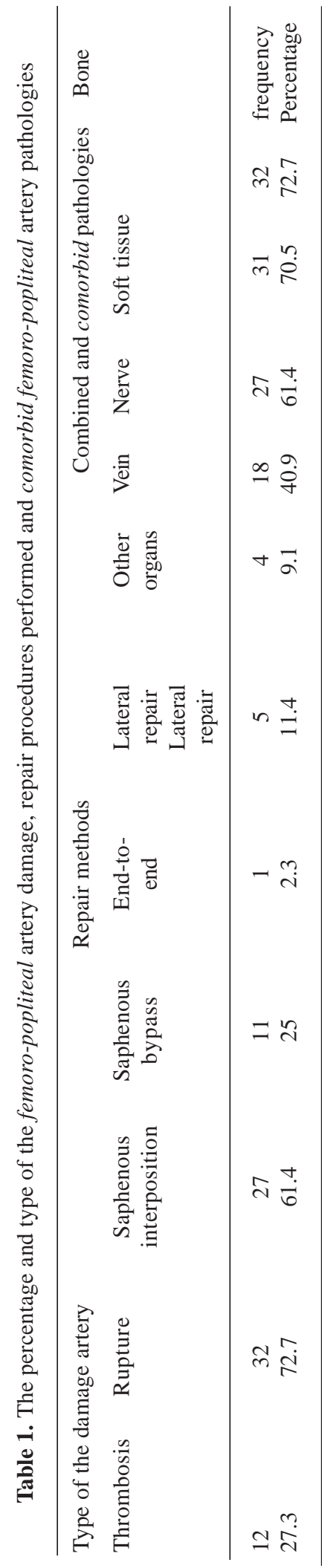


laceration and vascular trauma.

In this study, the percentage, and type of the femoro-popliteal artery damage, repair procedures performed and comorbid femoropopliteal artery pathologies were assessed that shown in the table 1.

As we see in the table the most common combined injuries in this study were respectively bone fractures and soft tissue damage and the most common vascular repair method performed was the saphenous vein graft interposition for the other foot and the most common type of artery injury was the femoro-popliteal arterial rupture.

Clinical examinations of the damaged foot in two times before and after the surgery indicate the success of the operation and recovery of the organ. After surgery of vascular repair, the majority of the patients in the study were suffering from the distal palpable pulse along with obvious pain reduction, normal c. f. and warmth of the organ.

Out of 44 patients studied 18 people (40.9\%) due to the obvious signs of compartment fasciotomy of compartment syndrome were suffered from the compartment of leg that 13 patients (72.2\%) were fully recovered after fasciotomy.

In this study the average time of ischemia (since the incident until reperfusion) was also investigated that has been shown in table 2 .

The independent t-test was used to compare the average time of ischemia (since the accident until reperfusion) among amputee and recovered persons as well as two groups that

Table 2. The average time of ischemia since the incident until reperfusion

\begin{tabular}{|c|c|c|c|c|}
\hline & & $\bar{X} \pm S D$ & Max & Min \\
\hline \multirow{3}{*}{$\begin{array}{l}\text { The average time of } \\
\text { ischemia (hour) }\end{array}$} & $\begin{array}{l}\text { Since the accident until the arrival } \\
\text { of the emergency }\end{array}$ & $13 \pm 15$ & 72 & 1 \\
\hline & $\begin{array}{l}\text { Since the arrival of the emergency } \\
\text { until the reperfusion }\end{array}$ & $12.5 \pm 22.2$ & 144 & 2 \\
\hline & $\begin{array}{l}\text { Since the accident until the } \\
\text { reperfusion }\end{array}$ & $26.1 \pm 24.6$ & 149 & 6 \\
\hline
\end{tabular}

Table 3. Compare the average time of ischemia among amputee and recovered persons as well as two groups

\begin{tabular}{cccc}
\hline & & $\bar{X} \pm S D$ & $P$-Value \\
\hline $\begin{array}{c}\text { The average time of } \\
\text { ischemia (hour) }\end{array}$ & $\begin{array}{c}\text { Recovered } \\
\text { Amputee }\end{array}$ & $26.6 \pm 26.4$ & \\
The average time of & Fasciotomy & $37 \pm 35.4$ & $\mathrm{P}=0.72$ \\
ischemia (hour) & No fasciotomy & $18.6 \pm 11.5$ & $\mathrm{P}=0.047$ \\
\hline
\end{tabular}

Table 4. Compression the status of combined damages of amputee patients with the rest of patients whose affected organ has been fully recovered

\begin{tabular}{lcccccc}
\hline & $\mathrm{N}$ & Bone fractures & Soft tissue & Nerve & Vein & Every 4 damages \\
\hline Full recovery & 39 & $27(69.2 \%)$ & $27(69.2 \%)$ & $23(59 \%)$ & $13(33.3 \%)$ & $2(5.1 \%)$ \\
Amputation & 5 & $5(100 \%)$ & $4(80 \%)$ & $4(80 \%)$ & $5(100 \%)$ & $4(80 \%)$ \\
P-value & - & 0.3 & 0.99 & 0.63 & 0.008 & 0.001 \\
\hline
\end{tabular}


underwent fasciotomy and did not undergo fasciotomy that has been shown in table 3 .

The average time of ischemia in two amputee and recovered groups did not show a significant difference; however, there was a significant difference between the group with and without fasciotomy surgery (Table 3). Furthermore, comparing the two averages shows a two-time increase in the average time of ischemia compared to the group that did not undergo the fasciotomy.

Of the 44 patients, 14 cases (31.8\%) have faced the infection and necrosis of the muscle after surgery that underwent the debridement and frequent wash and as a result of these measures nine patients (64.3\%) were recovered fully and the rest suffered a foot amputation.

The amount of recovery or amputation in 44 damaged patients was another subject that indicated 39 people (88.9\%) were recovered and only five patients $(11.4 \%)$ underwent the amputations.

In table 4 the status of combined damages of amputee patients has been compared with the rest of patients whose affected organ has been fully recovered, and as we see the vein damage ratio showed a significant difference only in two amputee and recovered groups $(\mathrm{p}=0.008)$.

In order to compare the mechanism of trauma in both groups: recovered (61.5\% with blunt mechanism) and amputee ( $80 \%$ with blunt mechanism) the Fisher exact test was used that results did not show a significant difference $(\mathrm{p}=$ $0.63)$.

\section{DISCUSSION}

This study was performed to evaluate the outcomes of femoropopliteal vascular trauma surgery in Ahvaz Golestan Hospital (Ahvaz, Iran). Of all patients, $86.4 \%$ were male and their average age was $27.7 \pm 12$ years. In a study by Melton $e t$ $a l .{ }^{11}$ approximately $86.3 \%$ of patients were male, as well. Mazrim et al.'s study ${ }^{12}$ showed that $86.5 \%$ of the injured persons were male with an average age of 34 years old that is almost similar to the present study. In a study by Philip et al. also $82 \%$ of the populations were male and the average age was 33 years. It is obvious that in all studies men are at risk more than women and most of the injured persons are young adults ${ }^{13}$.
This study showed that the long time average of ischemia (Since the accident until the reperfusion) alone cannot be a factor for the high incidence of amputation or failure to perform the vascular reconstruction. Although the average time of ischemia (accident to the reperfusion interval) was $26.1 \pm 24.6$, the vascular repair treated $88.6 \%$ of patients and only $11.4 \%$ of the injured persons were amputee (Table 2). In addition, multiplecombined damages including damage to the vein (which exists in all amputee patients) have an important role in amputation (Table 4). The analyses showed no statistically significant difference in the ratio of the damage to the vein between the two amputees and recovered groups.

On the other hand, the average time of ischemia in two amputees and recovered groups was did not show significant difference (Table 3).

It should be noted that one of main reasons for the prolongation of the average time of ischemia in the study was that $72.7 \%$ of the patients have been referred from the counties of Khuzestan Province and prolongation of hospitalization of the patient in the hospitals of these counties and distance from the center of the province causes prolongation of ischemia time. Justin et al. (2011) showed that ischemia time than six hours is not considered as a factor for predicting the limb amputations ${ }^{10}$, which is consistent with the present study. Although Dar et al. showed that reestablishing blood flow after $12 \mathrm{hrs}$ is associated with a high incidence of amputation.

The rate of amputation in our study was $11.4 \%$, while the rate of amputations after femoropopliteal vascular traumas has been reported up to $36 \%{ }^{15-17}$. In study by Saeed et al., $30 \%$ of the injured persons underwent amputation ${ }^{18}$ and in study by Davoodi et al. 34\% of patients have been amputated ${ }^{19}$ and this shows that amputations in our study compared to other similar studies has been at the possible lowest rate.

In this study, $40.9 \%$ of the injured persons became in need of fasciotomy that finally $72.2 \%$ of these people, who underwent a fasciotomy, were fully recovered and the injured limbs were preserved. Need to fasciotomy in patients with popliteal vascular damage has been reported up to $50 \%{ }^{10}$ that is consistent with Philip et al.'s study in which $49 \%$ of fasciotomy cases were necessary ${ }^{13}$. 
As we can see in table 3 , the average time of ischemia in both groups that underwent or did not undergo the fasciotomy shows a statistically significant difference $(\mathrm{P}=0.047)$ and comparison between the two means explains an almost twofold increase in the average time of ischemia in the group that underwent the fasciotomy compared to another group that did not undergo the fasciotomy. In our study $31.8 \%$ of patients were faced with post-operative infection and necrosis of the muscle as a result of the repeated washing and debridement of necrotic tissues $64.3 \%$ of patients fully recovered and the rest, who had severe and multiple combined damages such as venous trauma, were suffered by a foot amputation, which indicates an important role of frequent washing and debridement of necrotic tissue in recovering and maintaining the limb.

Among the combined injuries, only damage to the vein in both amputees and recovered groups showed a significant difference $(P=0.008)$ so that $100 \%$ of amputees have been suffered from venous injury and the recovered group had a venous trauma of 33.3\% (Table 4). This indicates an important role of venous trauma as the most important of combined injury of femoro-popliteal vascular trauma leading to the limb amputation.

By using Fisher exact test we evaluated companionship of every four combined injuries in two amputee and recovered groups that a significant difference was existed between the two groups ( $\mathrm{P}=0.001)$. As table 4 shows, $5.1 \%$ of recovered individuals had every four combined injuries, while $80 \%$ of amputees had every four combined injuries that this in turn indicates companionship of every four combined injuries of popliteal artery trauma in amputation of the injured limb.

At the same time, numerous combined injuries (more than one combined injury) should not hinder the vascular reconstruction. As the statistical survey in the study showed $84.6 \%$ of the recovered people had more than one combined injury along with femoro-popliteal artery trauma a combination would while in the amputee group $100 \%$ of injured people had more than one combined injure and Fisher exact test did not show a significant difference between the two groups.

\section{CONCLUSION}

Popliteal vascular injuries remains one of the most difficult diagnostic and therapeutic challenges for Vascular Surgeon, with rapid diagnosis and early interaction with meticulous technical skill can limb salvage be optimized. The long time average of ischemia alone cannot cause vascular remodeling or failure to perform an amputation. The multiple combined damages have important role in the amputation of a limb and damage to vein could have more important role. We recommend revascularization and fixation of bone and multistage debridement of limb and faciatomy if needed for all traumatic patients unless in limbs are irreversible in the clinical examination. In these cases initial amputation should be in mind. At the end, the need for early diagnosis at early referral to definitive care must be emphasized.

\section{ACKNOWLEDGEMENTS}

The financial support of this study was provided by Ahvaz Jundishapur University of Medical Sciences. We thankfully acknowledge the Department of Surgery, Golestan Hospital, Medical school of Ahvaz Jundishapur University of Medical Sciences. Our special thanks go to Molook Salemzadeh, the member of Golestan Hospital Clinical Development Research Unit for her contributions. Financial support of this study was provided by Ahvaz Jundishapur University of Medical Sciences.

\section{Authors' Contributions}

All authors contributed equally in planning and carrying out this project.

\section{Financial Disclosure}

The authors have no financial interests related to the material in the manuscript.

\section{REFERENCES}

1. Browner B.D, DeAngelis JP. Emergency care of Musculoskeletal Injuries, Sabiston textbook of surgery, 6th edition. Philadelphia: WB Saundres Company, 2008.

2. Frykberg ER. Popliteal vascular injuries. Surg Clin North Am. 2002;82: 67-89.

3. Iqbal khan M, Sayaf A, Hasan F.C. Evaluation 
of emergency revascular trauma. J Vasc Surg 2006; 33(6): 1212-1219.

4. Rasmussen TE, Clouse WD, Jenkins DH, Peck MA, Eliason JL, Smith DL. The use of temporary vascular shunts as a damage control adjunct in the management of wartime vascular injury. J Trauma. 2006; 61:8-15.

5. Todd R, Vase MD, Moral HL. Injuries to the peripheral Blood Vessels, chapter 17, ACS Surgery, 9th editon. New York: WebMD Professional Publishing, 2007.

6. Subramanian A, Vercruysse G, Dente Ch, Wyrzykowski A, Erin K, David VF. A decade's experience with temporary intravascular shunts at a civilian level I trauma center. J Trauma. 2008; 65:316-326.

7. Hafez HM, Woolgar J, Robbs JV. Lower extremity arterial injury: results of 550 cases and review of risk factors associated with limb loss.J Vasc Surg 2001; 33:1212-19.

8. Melton SM, Croce MA, Patton Jr JH, et al. Popliteal artery trauma. Systemic anticoagulation and intraoperative thrombolysis improves limb salvage. Ann Surg 1997; 225: 518-29.

9. Marshal JC, Meakins JL. Vascular injury in the leg, chapter 9, Emergency Vascular surgery. 1st editon. Springer, Berlin, Heidelberg, New York. 2007.

10. Simmons JD, Gunter JW, Schmieg Re, Manley JD, Rushton FW, Porter JM, Mitchell ME. Popliteal Artery injuries in an urban Trauma center with a Rural Catchment area: Do Delays in Definitive Treatment Affect Amputation? The AMERICAN Surgeon 2011; 77: 1521-1525.
11. Melton J, Sherry M. Popliteal Artery Trauma: Systemic Anticoagulation and Intraoperative Thrombolysis Improves Limb Salvage. Annals of surgery 1997; 225(5): 518-529.

12. Mazrim Y, Bibombep M, Manzoor A, Suhakara R, Kishore S. Popliteal artery injury: Royal perth experience and literature review. ANZ J Surg 2005; 75: 882-886.

13. Mullenix PS, Steels SR, Andersen CA. Limb salvage and outcomes among patients with traumatic popliteal vascular injury: An analysis of the National Trauma Data Bank. J Vasc Surg 2006; 44(1): 49-100.

14. Wagner WH, Calkins ER, Weaver FA, et al. Blunt popliteal artery trauma: one hundred consecutive injuries. J Vasc Surg 1988; 7:73643.

15. Harrell DJ, Spain DA, Bergamini TA, et al. Blunt popliteal artery trauma: a challenging injury. Am Surg 1997; 63: 228-31.

16. Yahya MM, Mwipatayi BP, Abbas M, et al. Popliteal artery injury: Royal Perth experience and literature review. ANZ J Surg 2005; 75:8826.

17. Bryan T, Merritt P, Hack B, et al. Popliteal arterial injuries associated with fractures or dislocations about the knee as a result of blunt trauma. Orthop Rev 1991; 20: 52530.

18. Journal of bone and joint surgery, Iran, volume IV, issue 3 (issue number 15), spring 2006, pages 21-17

19. Journal of Mazandaran University of medical sciences, volume 20, issue 79, December 2010, p. $31-38$ 